\title{
DC Railway Simulation Including Controllable Power Electronic and Energy Storage Devices
}

\author{
Pablo Arboleya, Senior Member, IEEE, Bassam Mohamed, Student Member, IEEE and Islam El-Sayed
}

\begin{abstract}
This work presents a comprehensive set of steady state models to be included in power flow simulation studies of DC railway networks. This simulation framework covers all important aspects and features of each element of modern DC railways. The proposed models are simplified to achieve the maximum simulation speed while keeping the required accuracy. Not only non-reversible, controlled and uncontrolled reversible substations are considered, but also on-board and off-board accumulation systems. The train model can consider the low network receptivity (overvoltage protection for trains equipped with regenerative braking) and overcurrent protection. It is also possible to include in the simulation DC/DC links between nodes of the railway network at the same or different voltage. To date, there is no other work able to conjugate all the mentioned models in a complex multi-train scenario.
\end{abstract}

Index Terms - catenary free, controlled substation, DC power systems, energy storage, multi-train, non-reversible substations, traction networks.

\section{INTRODUCTION}

D C traction systems are used to feed many types of railway transportation systems, from light trams at $750 \mathrm{~V}$, to metro systems or heavy trains at $1500 \mathrm{~V}$ or $3000 \mathrm{~V}$. The implementation of the existing technology in the electrical traction network and in the vehicles followed different paces. For instance, while the regenerative braking systems in the trains can be considered a mature technology, most of the DC traction power systems are fed using conventional noncontrolled diode based substations.

Traditionally, railway power system operators have been reluctant to include new technologies based on controlled power electronic devices and storage systems. It must be considered that low power quality and low efficiency were not a major concern, and diode based substations present very high reliability indices. Nowadays, the emergence of technologies based on controlable power electronic devices and storage systems is unstoppable due to the cost reduction and the need of increasing system efficiency, controllability and power quality.

One of the most used methodologies for planning and operation purposes in all kind of electrical networks is the power flow analysis. For this reason, the development of steady state simulation models considering all new technologies is a critical issue. In recent years many authors proposed new power flow algorithms and steady state models to include the new devices in the simulations. A complete simulation framework should be able to deal with all the typical modern DC railway power systems features listed below:

P. Arboleya, B. Mohamed and I. El-Sayed are with the Department of Electrical Engineering, University of Oviedo, Spain e-mail: arboleyapablo@uniovi.es

This work was partially supported by the Government of the Principality of Asturias under grant FC-15-GRUPIN14-127.

Manuscript received XXX, 2017; revised XXX, 2017.
- Complex multi-train topology.

- Non-reversible substations.

- Controlled/uncontrolled reversible substations.

- Off-board accumulation systems.

- On-board accumulation systems.

- Regenerative braking of the trains.

- Low network receptivity (train overvoltage protection).

- Train overcurrent protection.

- Combination of catenary free operation segments with conventional catenary lines.

- DC/DC links between nodes of the same voltage level or different voltage levels with different kind of control.

First power flow tools for DC trains simulation were proposed in the 70s [1]. Since very early developments, the regenerative braking of the trains was included in multi-train simulation tools [2]. Low network receptivity in high catenary voltage scenarios was not included in the models until the 90s [3], but in such cases, non-reversible and controlled substations models were not considered. During the last decade, the proliferation of these kind of tools, models, simulations and studies has suffered a drastic increase. In [4], a model with a very detailed simulation of the grounding system was proposed, but using ideal reversible substations. The work presented in [5] described a combined AC/DC power flow considering not only the DC railway system but also the AC distribution system. In this last case, the embedded substation and train models were very simplistic. Recently, many authors focused their efforts basically in two tasks:

- The simulation of the low network receptivity, like for instance the works presented in [6]-[11]. None of these works consider any kind of accumulation systems or hybrid catenary free and catenary feed networks.

- The development of on-board and off-board accumulation methods [12]-[15]. The studies presented in [12] do not include on-board accumulation, and in [13], [14], off-board accumulation is not considered. In [13] the piecewise definition of the functions was simplified using the Fischer-Burmeister approach to avoid the "if-then" implementation slowing down the solving procedure. The work in [15] is just applied to AC railway networks. In all cases, controllable substations are not considered.

Another group of authors are developing very detailed models of the different devices. Controlled substation models are proposed in [16], non-reversible substations in [17] and controlled substation plus off-board accumulators in [18]. In the case of trains, many references describing quite complex and accurate models [19]-[21] can be found. In [19], regenerative braking is not considered and in [21], on-board accumulation is not embedded in the model. In the cases where very detailed models are described, they are not applied to very complex multi-train scenarios. The main contribution 


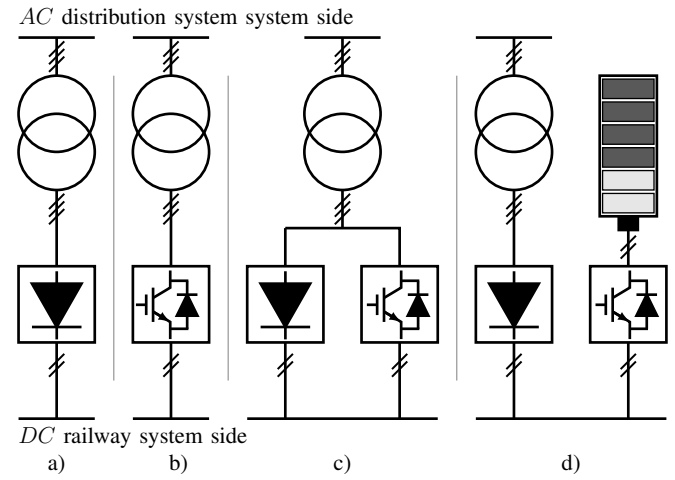

Fig. 1. Different types of railway substations. a) Conventional non-controlled diode type non-reversible substation. b) IGBT based reversible substation. c) Reversible substation with a diode rectifier in parallel with a IGBT based converter. a) Conventional non-controlled diode type non-reversible substation equipped with an off-board energy storage device

of the present work is the development of a comprehensive framework considering all the above described devices and features. The models are simple enough to be included in complex multi-train scenarios as it will be demonstrated in this paper, but also accurate enough to be used for planning and operation purposes. It must be emphasised that even when some of the models are not totally new, they provide a comprehensive and coherent framework for carrying out electrical simulations of DC-railways capturing the existing interactions. Heretofore, no other work or solver considers the wide range of models presented here at the same time.

The paper is structured as follows; the next section details all the proposed mathematical models. In section III, a description of the modified current injection (CI) power flow algorithm in which all the described models are embedded is presented. Section IV, has two subsections, the first one describes a realistic case study and some of the obtained results. In the second subsection, the convergence rate and the speed of the proposed algorithm will be analyzed and compared with other two well known algorithms like the Newton-Raphson (NR) based algorithm and the Backward Forward Swept (BFS) algorithm. The conclusions are presented in section $\mathrm{V}$.

\section{MATHEMATICAL MODEL}

\section{A. AC/DC substations}

In Fig. 1. different kind of substations connecting the AC distribution system with the DC traction system are represented. Traditionally, DC railway traction systems have been fed using diode based non-controlled substations (Fig. 17)). This technology is cost effective and robust. It can be also efficient in very dense traffic scenarios in which the probability of having trains injecting power and demanding power at the same time is very high. However, it is not efficient in scenarios with few trains and it is not controlable. The main drawback has to do with this last feature, since there is no way to return back to the AC network the energy regenerated by the trains during the braking process. One simple and effective way to overcome this problem is the use of IGBT based substations (Fig. 1p)). In this case, the substations are controllable in both directions, forward (AC to DC) and reverse (DC to AC). However, due to reliability reasons, the manufacturers prefer to keep the diode bridge rectifier for forward power flows

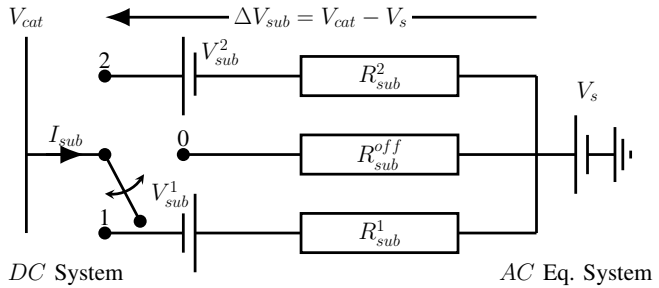

Fig. 2. Schematic representation of the substation general model. The left side represents the DC traction system, while the right side represent the equivalente AC distribution system modelled using a DC distributed slack bus. The current arrow represent the positive direction of the current

and connect an IGBT based converter in parallel for reverse power flows (Fig. 1. )). Usually, the rated power of the diode based rectifier is higher than the IGBT converter. It must be considered that the commutation losses of IGBT substations are higher than the GTO or diode ones. Another solution to improve voltage control in the DC traction system is based on the combination of conventional diode based substations with off-board accumulation systems (Fig. 11)). In most cases, the off-board accumulation systems are installed together with the substation, but in other cases they are installed in nodes of the traction network that are not directly connected to the AC distribution system. In this work, both possibilities are considered since the accumulation systems and the substations are modelled separately. In this subsection we will consider only the substation model representing the connection between the AC and the DC subsystems. In the next subsection the off-board accumulator model will be detailed. All the above described substations can be represented in a general model depicted in Fig. 2. $V_{s}$ is an input of the problem and it represents the $\mathrm{AC}$ equivalent voltage. The $\mathrm{AC}$ source is modelled as an equivalent DC distributed slack bus and its value can be different in each substation. $V_{\text {cat }}$ represent the catenary voltage and it is an output of the problem. It must be pointed out that for the sake of simplicity we refer to the DC traction system voltage as catenary voltage. However, it could also represent the third rail voltage in DC traction systems fed by this technology. The behaviour of the substation depends on the described variables since the three position switch represented in Fig. 2 is a voltage driven switch.

$$
\begin{aligned}
& \text { switch position }=f\left(\Delta V_{\text {sub }}\right) \\
& \text { where } \Delta V_{\text {sub }}=V_{\text {cat }}-V_{s}
\end{aligned}
$$

It must be considered that the use of a problem output $\left(V_{c a t}\right)$ in the switch control could create some convergence problems that in our case are overcome using a damping factor described in the next section. More information about how the use of problem outputs as control variables can create convergence problems can be found in [10], [11], [22]. Usually, the impedances in the branches 1 and 2 of the Fig. $2\left(R_{s u b}^{1}\right.$ and $\left.R_{s u b}^{2}\right)$ are calculated considering the voltage drop in the power transformer and the converter. Many authors used similar approaches to model railway substations [8], [11], [17]. Further information about detailed methods to calculate these impedances can be found in [11], [23], [24]. $R_{\text {sub }}^{\text {off }}$ is a high impedance used to improve the convergence performance when the substation is blocked. The voltage sources $V_{s u b}^{1}$ and $V_{s u b}^{2}$ are used to emulate the substation controls. Multiple kinds of controls and substations 


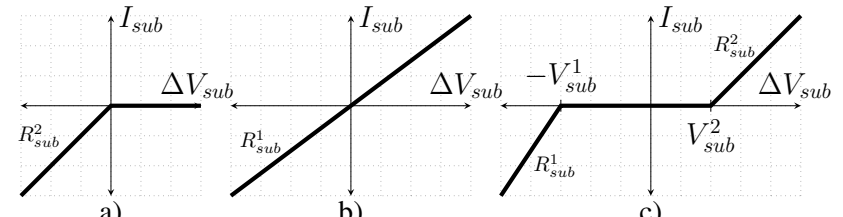

a)

b)

c)

Fig. 3. Substation model. a) Conventional non-reversible substation. b) Ideal reversible substation, c) Reversible substation with/without deadband and the posibility of considering different power converters for forward and reverse modes

technologies can be simulated using this general model, setting up correctly the above-mentioned parameters. In this section, three typical controls will be explained, but other different controls could be considered with the same general model. As it can be observed in Fig. 2, the substation current is positive when it is flowing from DC to AC. Thus, the forward power flow will imply negative current. The first set up to be considered represents the conventional diode based nonreversible substation like the one represented in Fig. 117). The characteristic of this substation is represented in Fig. 3 a). As it can be observed, the current is negative (forward power flow) if the slack voltage $\left(V_{s}\right)$ is higher than the catenary voltage $\left(V_{\text {cat }}\right)$ (negative $\left.\Delta V_{s u b}\right)$. Otherwise, the substation is blocked. In this case the switch will be in position 1 when the substation is conducting in forward mode and in position 0 when blocked. This behaviour is summarised in (2). It must be pointed out that in this case $V_{s u b}^{1}$ is set to 0 .

$$
I_{\text {sub }}=\left\{\begin{array}{llc}
\frac{\Delta V_{\text {sub }}}{R_{\text {sub }}^{1}} & \Delta V_{\text {sub }} \leq 0 & (\text { switch }=1) \\
0 & \Delta V_{\text {sub }}>0 & (\text { switch }=0)
\end{array}\right.
$$

The second configuration represent an ideal bidirectional substation that acts like an impedance conducting power in both directions (forward and reverse). This behaviour could be achieved with a substation equipped with an IGBT based converter like the one represented in Fig. 1 $1 \mathrm{~b}$ ). The characteristic of this control is represented in Fig. $3 \mathrm{~b}$ ). The switch will be always in position $1, V_{s u b}^{1}$ is set to zero and the current through the substation can be obtained using (3).

$$
I_{\text {sub }}=\frac{\Delta V_{\text {sub }}}{R_{\text {sub }}^{1}} \quad(\text { switch }=1)
$$

The third control characteristic is presented in Fig. 36). As it can be observed, there is a deadband in which the substation is blocked in both directions. The deadband is defined with the parameters $V_{s u b}^{1}$ and $V_{s u b}^{2}$. Outside this deadband, the substation can conduct in forward mode (switch position $=1$ ) or reverse mode (switch position $=2$ ) depending on the value of $\Delta V_{s u b}$. This control is summarised in (4). It must be remarked that defining the deadband with two parameters $\left(V_{s u b}^{1}\right.$ and $\left.V_{s u b}^{2}\right)$ different from zero implies the use of controlable technology for both directions (forward and reverse). In the case of the substation represented in Fig. 10), $V_{s u b}^{1}$ should be set to zero.

$$
I_{\text {sub }}=\left\{\begin{array}{lll}
\frac{\Delta V_{s u b}+V_{\text {sub }}^{1}}{R_{\text {sub }}^{1}} & \Delta V_{\text {sub }} \leq-V_{\text {sub }}^{1} & (\text { switch }=1) \\
0 & -V_{\text {sub }}^{1}<\Delta V<V_{\text {sub }}^{2} & (\text { switch }=0) \\
\frac{\Delta V_{s u b}-V_{\text {sub }}^{2}}{R_{\text {sub }}^{2}} & \Delta V_{\text {sub }} \geq V_{\text {sub }}^{2} & (\text { switch }=2)
\end{array}\right.
$$

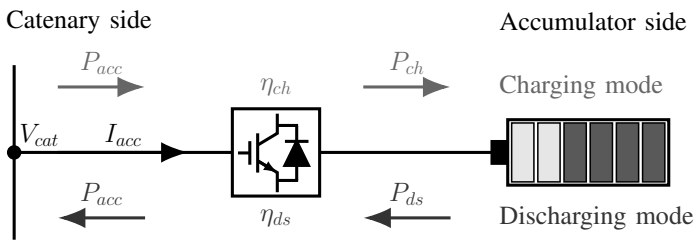

Fig. 4. Schematic representation of the off-board accumulator system. Variables on top of light grey arrows represent the charging mode while arrows on top of dark grey arrows represent the discharging mode. The current arrows represent the positive direction of the currents while the power arrows represent the actual direction of the power flow in both modes

\section{B. Off-board accumulators}

Off-board accumulator systems are a clear alternative to the substation reversibility and a good way to provide voltage support to the DC traction system. They are usually installed at the AC/DC substations with the configuration depicted in Fig. 1 1 ), but they can be also installed in other parts of the traction network. More information about the use of off-board accumulation systems for railway application can be found in [14], [18], [20], [25]. In this particular case, the off-board accumulator model is going to be described as an independent model. It can be connected to a substation node or any other traction node not connected to the AC system. The off-board accumulator is a voltage dependent power load/generator, the characteristic proposed in this work was already tested and validated by many authors for DC microgrids applications [26], [27]. It will act as a voltage support device, injecting power when the catenary voltage drops below a certain value, and absorbing power in case of high catenary voltage. The power injected or absorbed will depend on the catenary voltage as it was mentioned, but also on the state of charge of the accumulator as well as other set up parameters of the device.

In Fig. 4, the schematic representation of the accumulator is shown. The left side represent the catenary and the right side represent the accumulator device. Both parts are connected through a power converter. It is possible to assign two different efficiencies to the power converter in discharging mode $\left(\eta_{d s}\right)$ and charging mode $\left(\eta_{c h}\right)$. Actually, in these figures, not only the power electronic equipment efficiency is considered, but also the efficiency of the electro-chemical conversion process. Following the same criteria that was applied to the substations, the current and the power in the accumulator $\left(I_{a c c}\right.$ and $\left.P_{a c c}\right)$ will be positive from left to right (in this case in charging mode). While $P_{a c c}$ stands for the power that the accumulator inject or extract from the DC traction system, $P_{d s}$ and $P_{c h}$ represent actual power that reach the accumulation system in discharging and charging mode respectively. Two protections are considered to avoid accumulator deep discharge or overcharge. Thus, the actual available power that can be extracted from the accumulator $P_{d s}^{a v}$ depends on the maximum power in discharge mode $\left(P_{d s}^{\max }\right)$ but also on the state of charge $(S O C)$ of the accumulator and the protection parameters $s o c_{1}$ and $s o c_{2}$ as it can be observed in Fig. 5a). and (5).

$$
P_{d s}^{a v}= \begin{cases}0 & S O C<s o c_{1} \\ \frac{S O C-s o c_{1}}{s o c_{2}-s o c_{1}} \cdot P_{d s}^{\max } & s o c_{1} \leq S O C \leq \operatorname{soc}_{2} \\ P_{d s}^{\max } & S O C>s O c_{2}\end{cases}
$$




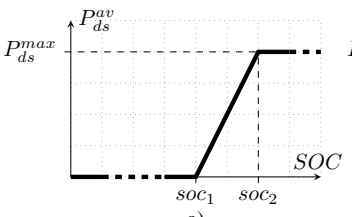

Fig. 5. a) Deep discharge protection characteristic. b) Overcharge protection

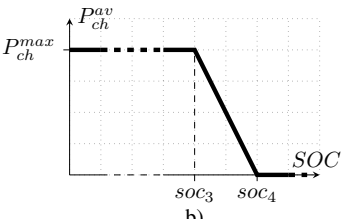

Fig. 5. a) Deep discharge protection characteristic. b) Overcharge protection characteristic

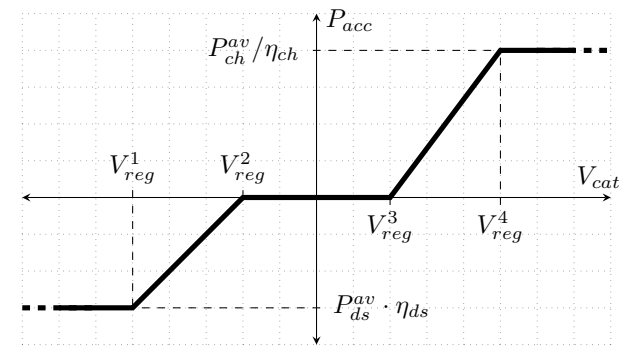

Fig. 6. Off-board accumulator system characteristic

In the same way, the overcharge protection can be formulated. The actual available power that can be injected into the accumulator $\left(P_{c h}^{a v}\right)$ depend on the maximum power in charge mode $\left(P_{c h}^{\max }\right)$ but also on the state of charge of the accumulator $(S O C)$ and the protection parameters $s_{0} c_{3}$ and $s o c_{4}$ as it can be observed in Fig. 5p). and (6).

$$
P_{c h}^{a v}= \begin{cases}P_{c h}^{\max } & S O C<s o c_{3} \\ \frac{S O C-s o c_{4}}{s o c_{3}-\operatorname{soc}_{4}} \cdot P_{c h}^{\max } & s_{0} \leq S O C \leq \operatorname{soc}_{4} \\ 0 & S O C>s o c_{4}\end{cases}
$$

The relation between the protection parameters must be the next one:

$$
\operatorname{soc}_{1}<\operatorname{soc}_{2}<\operatorname{soc}_{3}<\operatorname{soc}_{4}
$$

For each instant, and depending on the state of charge, the available power for discharging or charging the accumulator $\left(P_{d s}^{a v}\right.$ and $\left.P_{c h}^{a v}\right)$ can be computed before launching the power flow solver, since they do not depend on the solution of the problem. After that, the voltage dependent power characteristic of the accumulator can be build (see Fig. 6). The parameters $V_{\text {reg }}^{2}$ and $V_{\text {reg }}^{3}$ define the deadband in which the accumulator does not operate. The behaviour of the accumulator outside the deadband depends on the above described parameters as well as $V_{\text {reg }}^{1}$ and $V_{\text {reg }}^{4}$ as it can be observed in Fig. 6 and the expression (8) which describe the behaviour of the device.

$$
P_{a c c}= \begin{cases}-P_{d s}^{a v} \eta_{d s} & V_{c a t}<V_{r e g}^{1} \\ -P_{d s}^{a v} \eta_{d s} \frac{V_{r e g}^{2}-V_{c a t}}{V_{r e g}^{2}-V_{r e g}^{1}} & V_{r e g}^{1} \leq V_{c a t}<V_{r e g}^{2} \\ 0 & V_{r e g}^{2} \leq V_{c a t}<V_{r e g}^{3} \\ P_{c h}^{a v} / \eta_{d s} \frac{V_{c a t}-V_{r e g}^{3}}{V_{r e g}^{4}-V_{r e g}^{3}} & V_{r e g}^{3} \leq V_{c a t}<V_{r e g}^{4} \\ P_{c h}^{a v} / \eta_{c h} & V_{c a t} \geq V_{r e f}^{4}\end{cases}
$$

\section{DC/DC links}

The proposed framework can simulate the connection between two DC nodes with different voltage through a DC link. The general model proposed for DC/DC links is very flexible and it allows to implement many different operation modes representing different technologies. The DC link model is quite similar to the substation model as it can be observed comparing Fig. 2 (substation model) and Fig. 7 (link model). However, in the case of the link, there is not any slack voltage

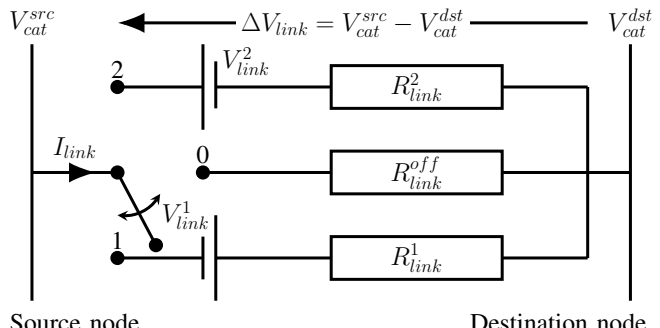

Source node

Destination node

Fig. 7. Schematic representation of the substation link model. The left side represents the source node and right side represent destination node, both of them in the DC traction system. The current arrow represent the positive direction of the current

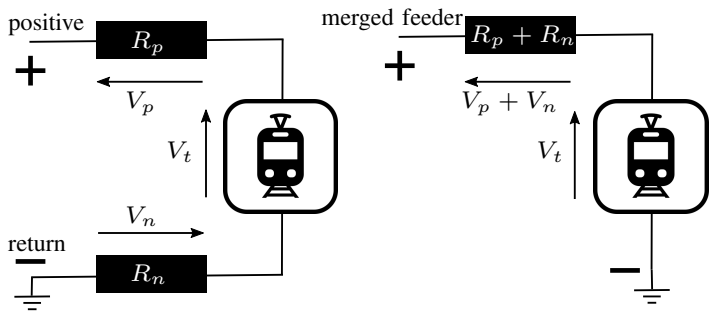

Fig. 8. Different approaches for modelling the lines. a) Positive and return conductores modelled separately. b) Simplified model grouping the positive and return conductors

source $\left(V_{s}\right)$. The switch position in the link model will depend also on the voltage drop through the link as it can be observed in the next expression.

$$
\begin{aligned}
& \text { switch position }=f\left(\Delta V_{\text {link }}\right) \\
& \text { where } \Delta V_{\text {link }}=V_{\text {cat }}^{s c}-V_{\text {cat }}^{d s t}
\end{aligned}
$$

As in the case of the substation model, three different configurations will be considered for the links. The conventional non-reversible link, the ideal bidirectional link and a reversible link with or without deadband. The current and the power in the link will be positive when flowing from source to destination. The three above-mentioned controls can be explained with (2), (3) and (4) and Figs. 3 a), b) and c) respectively, substituting the subindex "sub" by "link". It must be pointed out that with this definition, non-reversible links will transport power from destination node to source node and this power will be negative.

\section{D. line model}

Regarding the line model, the authors considered a simplified model in which the trains are connected directly to ground and fed by a conductor in which the positive and return impedances are represented. This approach is widely accepted [10]-[12], [28]. The other approach is based on considering the positive and return conductors as separate circuits [7], [8]. The latter can be more accurate, if a detailed model of the track is used and it allows to handle the voltage drops in the contact system and the return system separately; however the former is computationally lighter. When an accurate calculation of the rail potential or stray currents is needed, the second approach is the only option [29], [30]. However, for power flow purposes, the results obtained with the first approach are acceptable when compared with experimental results [14]. Both approaches are represented in Fig. 8. The authors of this work consider other two possibilities for catenary-free 


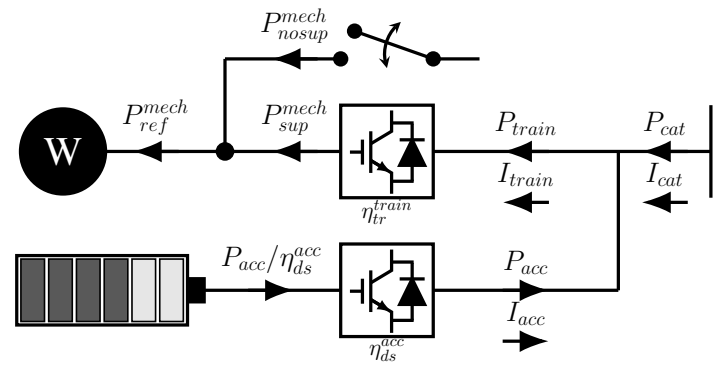

Fig. 9. Train model in traction mode. The current arrows represent the positive direction of the current while the power arrows represent the actual direction of the power flow

segments. The first one is that even when the train is not connected to the catenary, the positive feeder is buried, so the two nodes adjacent to a catenary-free segment will be electrically connected. This kind of line will be labelled as catenary-free connected line. In the second possibility, there is no electrical connection between the nodes if the segment between them is catenary-free. The second option will be labeled as catenary-free disconnected line. In both cases the train will be fed by its on-board accumulation system.

\section{E. Train model}

The train is the most complex device in the network and it is simulated in a decoupled way. That means that the train mechanical power reference and its position are provided by an external software and those data are used as inputs for the network solver. This approach is widely accepted [7], [8], [11]. It must be remarked that with the decoupled approach, the driving and the position of the trains are predefined before the simulation starts and they cannot be altered due to the conditions of the network. However, even when the decoupled method is selected, the influence of the catenary voltage over the final injected or demanded power is considered by means of the overcurrent and overvoltage protection, so it is possible to warn the designer/operator in the event that the train can not obtain the required power from the network. In [8], the overvoltage protection is modelled and [11] considers also the overcurrent protection. None of these references include the on-board accumulation system coordinated with the abovementioned train model. The on-board accumulation system is modelled as a separate device that travels together with the train. During the solving procedure, train and accumulator are coupled in order to simulate the accumulator control. The control philosophy assigns priority to the accumulator. When the train accelerates, it uses the maximum available power from the accumulator. When it brakes, the accumulator system tries to absorb all the regenerated power if it is possible. The best way to explain the train and on-board accumulator coupled models is to assume that the catenary voltage $\left(V_{c a t}\right)$ is known, which is true for a given iteration of the power flow calculation.

1) Train in traction mode and on-board accumulator in discharging mode: The train plus the accumulator in traction mode is represented in Fig. 9. In this figure, the currents $I_{\text {train }}$, $I_{a c c}$ and $I_{c a t}$ represent the current absorbed by the train, the current injected by the accumulator and the current absorbed from the catenary respectively. These currents determine also the positive reference for the powers. As it can be observed, all powers are positive in this case. The characteristic of the train in traction mode is represented in Fig. 10 a). The parameters $V_{\text {train }}^{1}$ and $V_{\text {train }}^{2}$ represent the train overcurrent protection and are fixed for a given train model. The train characteristic is multiplied at each instant by the mechanical reference power and divided by the train efficiency in traction mode $P_{r e f}^{\text {mech }} / \eta_{t r}^{\text {train }}$ obtaining the train characteristic equation that can be expressed as follows:

$$
P_{\text {train }}= \begin{cases}0 & V_{\text {cat }}<V_{\text {train }}^{1} \\ \frac{V_{\text {cat }}-V_{\text {train }}^{1}}{V_{\text {train }}^{2}-V_{\text {train }}^{1}} \frac{P_{\text {ref }}^{\text {mech }}}{\eta_{\text {tr }}^{\text {train }}} & V_{\text {train }}^{1} \leq V_{\text {cat }} \leq V_{\text {train }}^{2} \\ P_{\text {ref }}^{\text {mech }} / \eta_{\text {tr }}^{\text {train }} & V_{\text {cat }}>V_{\text {train }}^{2}\end{cases}
$$

It must be mentioned that the linear derating of the power is an approximation since the real model should consider a linear derating of the current, what means a quadratic variation of the power like the one proposed in [13]. However, this definition is much simpler and the results are accurate enough for power flow purposes as it was expressed in [14] where a similar train model is used and validated with experimental results.

For a given catenary voltage $\left(V_{\text {cat }}\right)$, the train power $\left(P_{\text {train }}\right)$ is calculated according to $(10)$. The real mechanical power supplied to the train $\left(P_{\text {sup }}^{\text {mech }}\right)$ is obtained using the train efficiency in traction mode by means of the next expression:

$$
P_{\text {sup }}^{\text {mech }}=P_{\text {train }} \cdot \eta_{\text {tr }}^{\text {train }}
$$

If the catenary voltage is lower than $V_{\text {train }}^{2}$, there is nonsupplied power $P_{\text {nosup }}^{\text {mech }}$ that is calculated as

$$
P_{\text {nosup }}^{\text {mech }}=P_{\text {ref }}^{\text {mech }}-P_{\text {sup }}^{\text {mech }}
$$

As it was mentioned before, the train and the electrical network are modelled in a decoupled way [7], [8], [11]. For this reason, the position of the train at each instant is determined from the beginning of the simulation. In some cases, when the train is accelerating but the network cannot provide the total requested power due to the overcurrent protection. The difference between the reference power and the actual power absorbed from the catenary is labelled as non-supplied power. The train position in the next instant will be the scheduled, but the designer/operator will get a warning (the train at that specific instant cannot accelerate at the desired rate). Regarding the accumulator, it is defined by the deep discharge protection characteristic, as in the case of the off-board device (see Fig. 5 a) and (5)). In this case, $P_{d s}^{a v}$ represents the power available for discharging in the left side of the accumulator converter (see Fig. 9). The actual power injected in the train by the accumulator is calculated as:

$$
P_{a c c}=\min \left(P_{d s}^{a v} \cdot \eta_{d s}^{a c c}, P_{\text {train }}\right)
$$
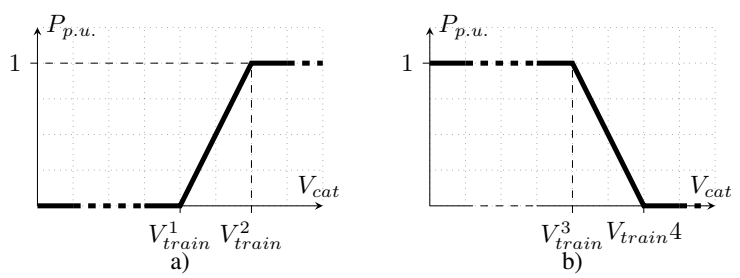

Fig. 10. Train characteristics in a) traction mode and b) braking mode 


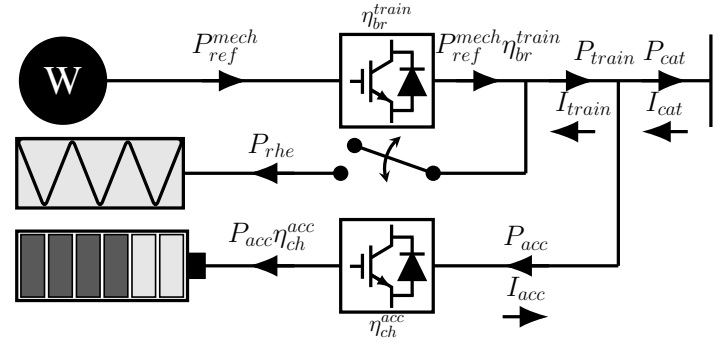

Fig. 11. Train model in braking mode. The current arrows represent the positive direction of the current while the power arrows represent the actual direction of the power flow

The power absorbed from the catenary is:

$$
P_{\text {cat }}=P_{\text {train }}-P_{\text {acc }}
$$

2) Train in braking mode and on-board accumulator in charging mode: This case is represented in Fig. 11. Again, the currents represent the positive references, as it can be observed, all powers will be negative in this case. The train characteristic in braking mode (see Fig. 10 b)) defines the overvoltage protection and the parameters $V_{\text {train }}^{3}$ and $V_{\text {train }}^{4}$ are fixed once the train model is defined. At each instant, the train characteristic is multiplied by the negative power reference and the train efficiency in braking mode $\left(P_{r e f}^{\text {mech }}\right.$. $\left.\eta_{b r}^{\text {train }}\right)$ obtaining the train characteristic equation in braking mode:

$P_{\text {train }}= \begin{cases}P_{\text {ref }}^{\text {mech }} \cdot \eta_{\text {train }}^{\text {train }} & V_{\text {cat }}<V_{\text {train }}^{3} \\ \frac{V_{\text {train }}^{4}-V_{\text {cat }}}{V_{\text {train }}^{4}-V_{\text {train }}^{3}} P_{\text {ref }}^{\text {mech }} \eta_{\text {br }}^{\text {train }} & V_{\text {train }}^{3} \leq V_{\text {cat }} \leq V_{\text {train }}^{4} \\ 0 & V_{\text {cat }}>V_{\text {train }}^{4}\end{cases}$

The characteristic that defines the on-board accumulator overcharging protection is the same that was previously defined for the off-board device (see Fig. 5 b) and (6). $P_{c h}^{a v}$ represents the power available for charging in the left side of the accumulator converter (see Fig. 11p. The actual power used in the right side can be calculated as:

$$
P_{a c c}=\max \left(P_{c h}^{a v} / \eta_{d s}^{a c c}, P_{\text {train }}\right)
$$

It must be remarked that all powers in (16) are negatives. The power absorbed from the catenary can be calculated using (14). The power burned in the rheostatic system $\left(P_{r h e}\right)$ is obtained using the next expression:

$$
P_{\text {rhe }}=P_{\text {ref }}^{\text {mech }} \cdot \eta_{b r}^{\text {train }}-P_{\text {train }}
$$

\section{CURRENT INJECTION POWER FLOW ALGORITHM}

The algorithm implemented for accommodating all the above proposed models is based on the well known current injection algorithm [11]. This kind of algorithm, as well as the Backward/Forward Swept algorithms use a formulation based in the Current Kircchoff Law (KCL) and Voltage Kircchoff Law (KVL) [10], [31]-[33] instead of the traditional power injection formulation used for instance by the conventional Newton-Raphson methods [8], [34]. The latter are usually faster for solving unconstrained power flow problems with smooth characterstics devices, but the former are faster and more stable for solving constrained power flow problems containing devices with non-smooth characteristics as it will be demonstrated later in this paper. In Alg. 1 the procedure for solving the power flow problem is summarised. The algorithm needs the set-up of the distributed slack voltage sources $\mathbf{v}_{\mathbf{s}}$ and the configuration parameters of the substations, off-board accumulators, links, lines, trains and on-board accumulators $\left(\mathbf{v}_{\mathbf{s}}, \mathbf{p}_{\text {subs }}, \mathbf{p}_{\text {off_acc }}, \mathbf{p}_{\text {links }}, \mathbf{p}_{\text {lines }}, \mathbf{p}_{\text {trains }}, \mathbf{p}_{\text {on_acc }}\right)$. Trains schedule can be set up with the parameters in $\mathbf{p}_{\text {sch }}$.

The first two steps are respectively the initialisation of the nodal voltage vector $\left(\mathbf{v}_{\mathbf{N}}\right)$ and the load current vector $\left(\mathbf{i}_{\mathbf{L}}\right)$. The load current vector includes all the currents of the offboard accumulation devices, on-board accumulation devices and trains. The function " $g()$ " obtains the current as a function of the voltage using the power-vs-current curves described section II. That means that for an specific device, let us say a train, depending on the catenary voltage, a power is calculated using the model described in Fig. 10 Once the power is obtained, it is divided by the nodal voltage in order to obtain the current. All this process is summarised in the step 2 of the algorithm by means of the function " $g()$ ". As it can be observed, the use of power-vs-voltage models was adopted in this work; nevertheless, the same algorithm can accommodate current-vs-voltage models. For initialisation purposes, a function " $g^{0}()$ " different from " $g()$ " could be used. After this step, all train currents, off-board accumulator currents and on-board accumulator currents are computed for the specific voltage profile at this iteration. In the next steps (3 and 4), two vectors containing the voltage drop across all substations and the links $\left(\Delta \mathbf{V}_{\text {sub }}\right.$ and $\left.\Delta \mathbf{V}_{\text {links }}\right)$ are computed using the initial voltage profile $\left(\mathbf{v}_{\mathbf{N}}\right)$, and once this voltage drop is calculated the switches positions in all substations and links ( $\mathbf{s}_{\text {sub }}^{\text {switch }}$ and $\left.\mathbf{s}_{\text {links }}^{\text {switch }}\right)$ are obtained in the steps 5 and 6 of the algorithm. With all the parameters defining the substations and the links and the position of the switches, the current through all of these devices ( $\mathbf{I}_{\text {sub }}$ and $\mathbf{I}_{\text {link }}$ ) can be computed in steps

\footnotetext{
Input: $\quad \mathbf{v}_{\mathbf{s}}, \mathbf{p}_{\text {subs }}, \mathbf{p}_{\text {off_acc }}, \mathbf{p}_{\text {links }}, \mathbf{p}_{\text {lines }}, \mathbf{p}_{\text {trains }}, \mathbf{p}_{\text {on_acc }}, \mathbf{p}_{\text {sch }}$ Output: $\mathbf{v}_{\mathbf{N}}, \mathbf{i}_{\mathbf{N}}, \mathbf{i}_{\mathbf{L}}$

1. SET all $\mathbf{v}_{\mathbf{N}}(k)=1 \leftarrow$ Nodal voltages init.

2. COMP all $\mathbf{i}_{\mathbf{L}}=g^{0}\left(\mathbf{v}_{\mathbf{N}}\right) \leftarrow$ Load currents init.

3. COMP all $\Delta \mathbf{V}_{\text {sub }} \leftarrow$ Voltage drops in substations

4. COMP all $\Delta \mathbf{V}_{\text {link }} \leftarrow$ Voltage drops in DC/DC links

5. COMP all $\mathbf{s}_{\text {sub }}^{\text {switch }}=f\left(\Delta \mathbf{V}_{\text {sub }}\right) \leftarrow$ Subs. switches

6. COMP all $\mathbf{s}_{\text {link }}^{\text {switch }}=f\left(\Delta \mathbf{V}_{\text {link }}\right) \leftarrow$ Links switches

7. COMP all $\mathbf{I}_{\text {sub }}=f\left(\Delta \mathbf{V}_{\text {sub }}, \mathbf{s}_{\text {sub }}^{\text {switch }}\right) \leftarrow$ Subs. curr.

8. COMP all $\mathbf{I}_{\text {link }}=f\left(\Delta \mathbf{V}_{\text {sub }}, \mathbf{s}_{\text {link }}^{\text {switch }}\right) \leftarrow$ Links curr.

9. COMPUTE all $\mathbf{i}_{\mathbf{N}} \leftarrow$ Nodal currents computation

10. COMPUTE $\mathbf{Y} \leftarrow$ System admittance matrix

11. $\mathbf{i}_{\mathbf{L}}^{0}=\mathbf{i}_{\mathbf{L}}$

12. $\mathbf{v}_{\mathbf{N}}^{0}=\mathbf{v}_{\mathbf{N}}$

13. $\mathbf{v}_{\mathbf{N}}=\mathbf{Y}^{-1} \cdot \mathbf{i}_{\mathbf{N}} \leftarrow$ Nodal voltages updating

14. $\mathbf{v}_{\mathbf{N}}=\alpha \mathbf{v}_{\mathbf{N}}^{0}+(1-\alpha) \mathbf{v}_{\mathbf{N}} \leftarrow$ Damping factor

15. $\mathbf{i}_{\mathbf{L}}=g\left(\mathbf{v}_{\mathbf{N}}\right) \leftarrow$ Load currents updating

16. IF $\left\|\mathbf{i}_{\mathbf{L}}^{0}-\mathbf{i}_{\mathbf{L}}\right\|>\varepsilon$ or $\left\|\mathbf{v}_{\mathbf{N}}^{0}-\mathbf{v}_{\mathbf{N}}\right\|>\varepsilon \quad$ GOTO 3

17. ELSE Break
}

Algorithm 1: Summary of the power flow algorithm 
7 and 8. By combination of all the above computed currents, load currents and substation and link currents, the vector with the nodal current injections $\left(\mathbf{i}_{\mathbf{N}}\right)$ can be obtained (step 9). The step 10, represent the admittance matrix calculation, it must be remarked that it must be computed each iteration because the state of the switches of the substations and links can vary from one iteration to the next one affecting the admittance matrix. As it will be demonstrated in this work, this is not a problem in terms of the speed of the solver. The load current and nodal voltage vectors of the previous iteration is saved in $\mathbf{i}_{\mathbf{L}}^{0}$ and $\mathbf{v}_{\mathbf{N}}^{0}$ in steps 11 and 12 before the new voltage vector is calculated in step 13 using the admittance matrix and the nodal current vector. A damping factor $(\alpha)$ is applied to the node voltage vector in the step 14 in order to update the voltage. As it will be demonstrated, the selection of this damping factor is critical in terms of the convergence rate of the algorithm. Once the new voltages are obtained, the load currents (train and accumulator currents) are updated by means of the described models. The updating of load currents, which depend on the voltage profile, is summarised by means of the function " $g()$ " in step 15 of the pseudocode. The current and voltage vectors obtained in the new iteration are compared with the stored ones from the previous iteration in the step 15. The convergence is achieved when the norms of the two difference vectors are below a given threshold defined by $\epsilon=10^{-5}$ in our case. If any of these norms are over the threshold, the admittance matrix considering the new voltage scenario is recalculated and the algorithm is executed again until convergence is achieved. For the sake of simplicity, it is not represented in the pseudocode, but a maximum number of iterations is also defined. If this number is reached the algorithm stops with an error message.

\section{Case Study and Algorithm Performance ANALYSIS}

All models have been tested separately and compared with real measurements. Regarding the power flow algorithm, extensive random test sets have been carried out to study the convergence. This section is divided in two parts. In the first subsection, a realistic case study is described, solved and analysed. In the next subsection, the algorithm performance is analysed for the described case study and a variation of this case, and it is compared in terms of speed and convergence rate with other well known algorithms like Newton-Raphson (NR) and Backward/Forward Swept (BFS).

\section{A. Case Study}

The case study is represented in Fig. 12. It contains 4 different lines, each of them with 6 substations. There are two different voltage levels, $1500 \mathrm{~V}$ for lines blue and red and $3000 \mathrm{~V}$ for lines green and orange. The blue and red lines have the first two substations in common. In the blue line, the tracks cover only the path from substations S1 to S4. However, S4 is electrically connected through a conventional electrical line to S5 and S6 (both in grey). This last substation (S6) is connected by means of two links with S5 in the orange line and S5 in the red line. The DC connection with the orange line is unidirectional with a deadband of $10 \mathrm{~V}$ referred to 1500

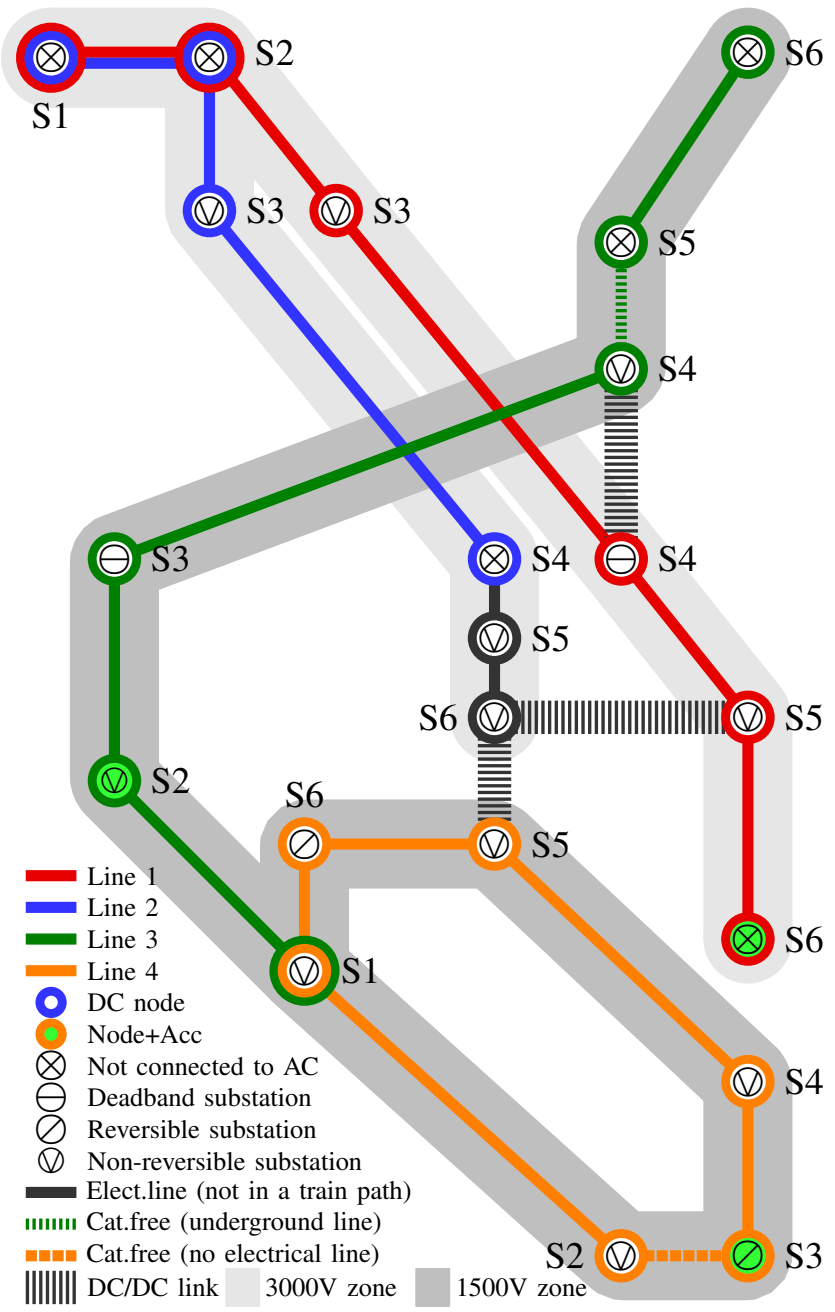

Fig. 12. Schematic representation of the case study

V. It will only transport power from the orange line to the blue line. The second link is an ideal bidirectional link connecting red and blue lines. There is a third link connecting S4 in the green line to S4 in the red line; it is a bidirectional link with deadband. The node in the green line is the source node and $V_{\text {link }}^{1}$ and $V_{\text {link }}^{2}$ are set to $20 \mathrm{~V}$ and $40 \mathrm{~V}$ respectively, referred to the source node voltage. There are two catenary free segments, the first one (between S4 and S5 in the green line) contains an electrical underground line, but the trains are not fed from it. In the second case (between S2 and S3 in the orange line), there is no electrical connection.

There are three off-board accumulator systems in substations S2 (green line), S3 (orange line) and S6 (red line). The three of them are $2 \mathrm{kWh}$ ultracapacitor based devices with a maximum charge and discharge power of $500 \mathrm{~kW}$ and an efficiency of 0.95 in charging and discharging mode. The $s o c$ parameters to set up the deep-discharge and overcharge protection are set to $10 \%, 20 \%, 90 \%$ and $100 \%$. The regulation voltages $V_{\text {reg }}^{1}, V_{\text {reg }}^{2}, V_{\text {reg }}^{3}, V_{\text {reg }}^{4}$ are $0.98,0.99,1.01$ and 1.02 p.u., respectively.

All lines in the system have a positive feeder resistance of $28.605 \mathrm{~m} \Omega / \mathrm{km}$, the rail resistance is set to $7 \mathrm{~m} \Omega / \mathrm{km}$. The total length of red and orange lines is $30.84 \mathrm{~km}$. The length of the blue and green lines is $36.93 \mathrm{~km}$. The lengths of the segments between the substations in the four lines can be found in Table II. The resistances $R^{1}$ and $R^{2}$ have been set in 
TABLE I

LENGTHS BETWEEN SUBSTATIONS IN KM

\begin{tabular}{c|c|c|c|c} 
& Red & Blue & Green & Orange \\
\hline \hline S1 - S2 & 4.316 & 4.316 & 8.120 & 7.310 \\
\hline S2 - S3 & 0.500 & 25.284 & 2.880 & 4.200 \\
\hline S3 - S4 & 13.800 & 7.335 & 12.560 & 2.600 \\
\hline S4 - S5 & 7.848 & 12.497 & 5.440 & 8.100 \\
\hline S5 - S6 & 4.378 & 19.797 & 7.930 & 3.700 \\
\hline S6 - S1 & $X$ & $X$ & $X$ & 4.930
\end{tabular}

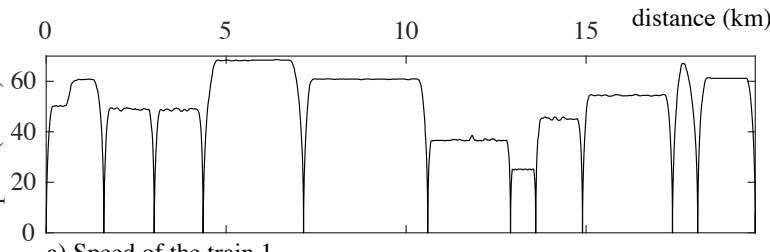

$$
\text { a) Speed of the train } 1
$$
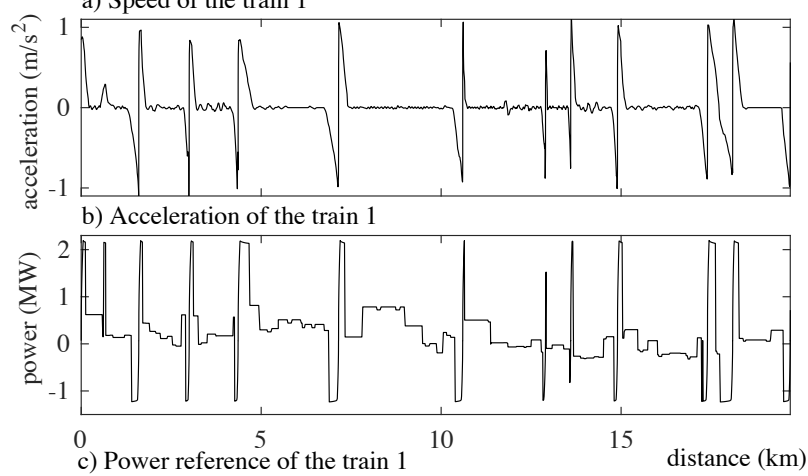

Fig. 13. Subfigures a), b) and c) represent, respectively, the speed, the acceleration and the power reference of the first train departing from Substation S1 to S6 (Green line) during the first 30 minutes of simulation

all links and substations to $270 \mathrm{~m} \Omega$ and $180 \mathrm{~m} \Omega$, respectively. In unidirectional substations $V_{s u b}^{1}$ has been set to $0 \mathrm{~V}$. In biderectional substations with deadband $V_{s u b}^{1}=V_{s u b}^{2}=20 \mathrm{~V}$.

There are 80 trains present in the system departing at 10 minutes intervals from all starting and ending points of all lines. In the case of the circular line (orange), the trains depart from S1 and make the path in clockwise and counterclockwise directions. All trains are equipped with an energy storage device based on ultracapacitors. The soc parameters and the efficiencies for the on-board accumulator are the same as the ones used for the off-board accumulators. The maximum charge and discharge power is $200 \mathrm{~kW}$ and the energy storage capacity is $5 \mathrm{kWh}$.

In Figs. 13 and 14 , some results obtained from the abovedescribed simulation are presented for the first half and hour of simulation. Fig. 13 contains the speed, acceleration and power reference of the train 1 in the green line as a function of the distance from substation 1 . The maximum speed of the train is reached between the stops 5 and 6 and it is $68.4 \mathrm{~km} / \mathrm{h}$ (the maximum allowed speed in that segment is $70 \mathrm{~km} / \mathrm{h}$ ). The acceleration is limited to $1.1 \mathrm{~m} / \mathrm{s}^{2}$ and the power ranges from 2.2 MW (maximum traction power) to $-1.25 \mathrm{MW}$ (maximum braking power). The trains in the rest of the lines have similar values.

The behaviour of the substation S2 in the green line, equipped with an off-board accumulator is represented in Fig. 13 a) and b). This substation is a unidirectional substation with a deadband of 10 V. As it can be observed in Fig. 13 a), the power provided by the substation (in black) is always negative
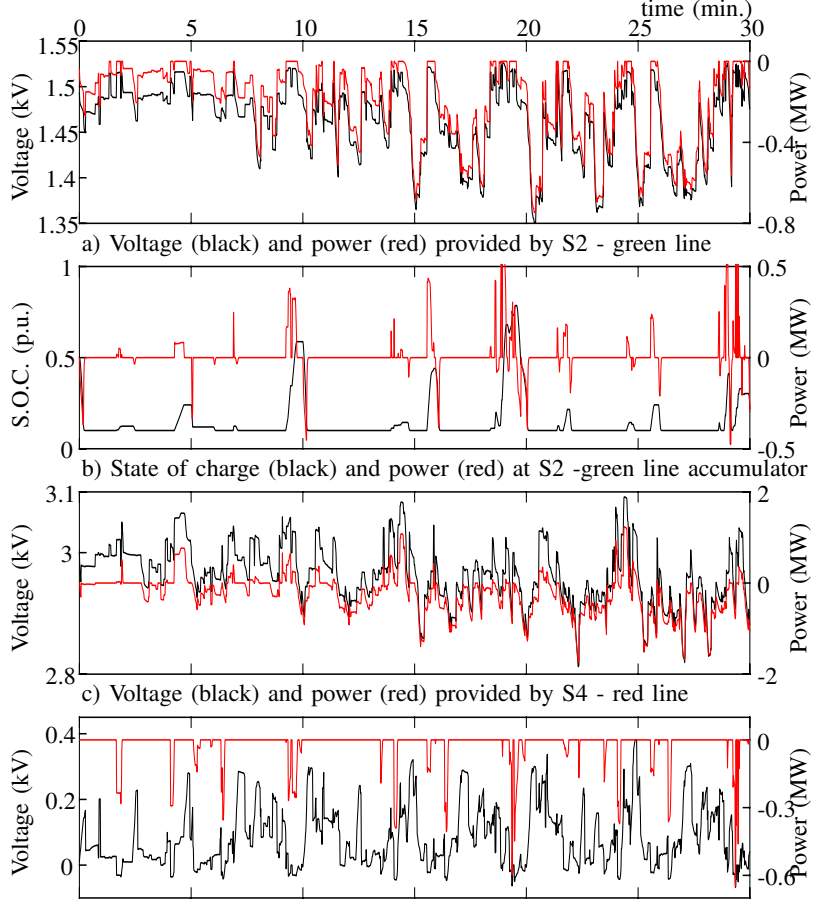

d) Voltage across the link S6 Blue - S5 Orange (black) and power (red)

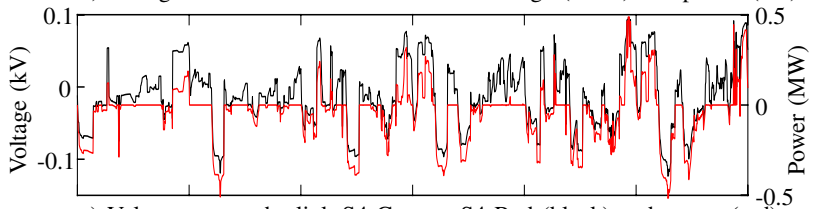

e) Voltage across the link S4 Green - S4 Red (black) and power (red)
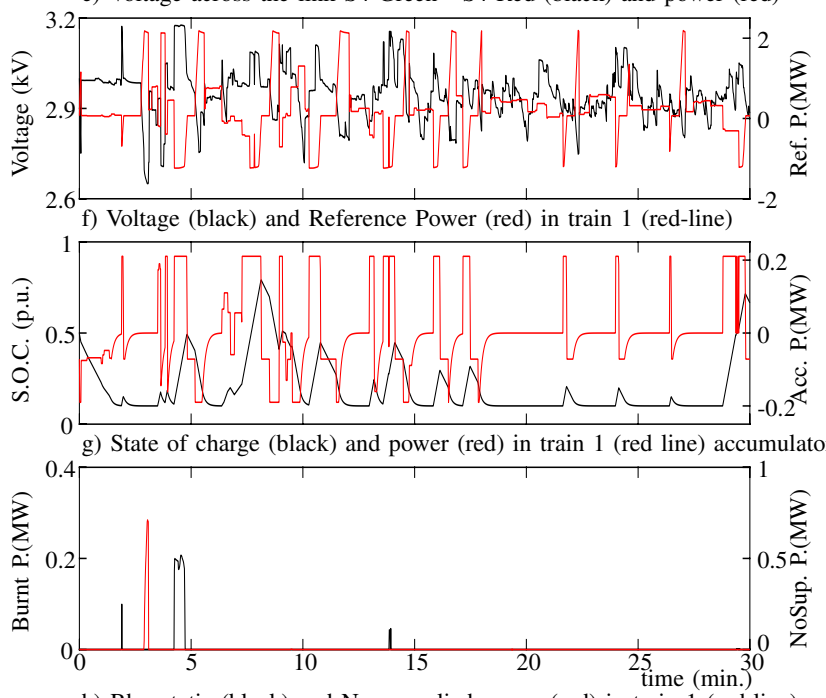

h) Rheostatic (black) and Non-supplied power (red) in train 1 (red-line)

Fig. 14. Case study results

or zero. In the instants where the DC voltage (in red) is higher than $1490 \mathrm{~V}$ the substation is blocked. When the voltage in the substation increases above $1515 \mathrm{~V}$, the substation accumulator charge is activated as it can be observed in Fig. 13 b). The discharging mode is activated when the voltage drops below $1485 \mathrm{~V}$. The state of charge of the accumulator never goes below 0.1 p.u. or increase above 1 p.u due to the deepdischarge and overcharge protection.

Substation S4 in red line is a deadband bidirectional substation. Its voltage and power are represented in Fig. 13 c). It only transport power from AC to DC (negative power) when the voltage drops below $1480 \mathrm{~V}$. The reverse mode is activated when the voltage increases above $1520 \mathrm{~V}$. 
TABLE II

ANALYSIS OF THE DAMPING FACTOR EFFECT OVER THE SPEED AND CONVERGENCE RATE OF THE PROPOSED CURRENT INJECTION ALGORITHM

\begin{tabular}{c|c|c|c|c|c|c|c|c|c|c} 
Damping factor & 0.1 & 1,125 & 0.15 & 0.2 & 0.3 & 0.325 & 0.5 & 0.6 & 0.65 & 0.7 \\
\hline \hline Min Time (ms) & 0.014 & 0.019 & 0.020 & 0.019 & 0.017 & 0.022 & 0.026 & 0.035 & 0.039 & $\mathrm{X}$ \\
\hline Max Time (ms) & 0.044 & 0.045 & 0.048 & 0.060 & 0.037 & 0.042 & 0.142 & 0.113 & 0.058 & $\mathrm{X}$ \\
\hline Avg. Time (ms) & 0.029 & 0.030 & 0.027 & 0.030 & 0.027 & 0.030 & 0.036 & 0.043 & 0.044 & $\mathrm{X}$ \\
\hline Solved cases (\%) & 80.89 & 87.22 & 100 & 100 & 100 & 99.940 & 99.330 & 99.170 & 56.390 & 0
\end{tabular}

TABLE III

COMPARISON BETWEEN THE THREE ALGORITHMS (NR, BFS AND CI)

\begin{tabular}{l|c|c|c|c|c|c} 
Case & \multicolumn{3}{|c|}{ All bidirectional (B) } & \multicolumn{3}{c}{ Real Case (A) } \\
\hline Algorithm & NR & BFS & CI & NR & BFS & CI \\
\hline \hline Min.Time (ms) & 0.089 & 0.005 & 0.010 & 0.137 & 0.017 & 0.019 \\
\hline Max.Time (ms) & 0.409 & 0.102 & 0.394 & 1.312 & 0.279 & 0.060 \\
\hline Avg.Time (ms) & 0.151 & 0.013 & 0.027 & 0.270 & 0.043 & 0.030 \\
\hline Solved(\%) & 100 & 100 & 100 & 90.888 & 63.277 & 100
\end{tabular}
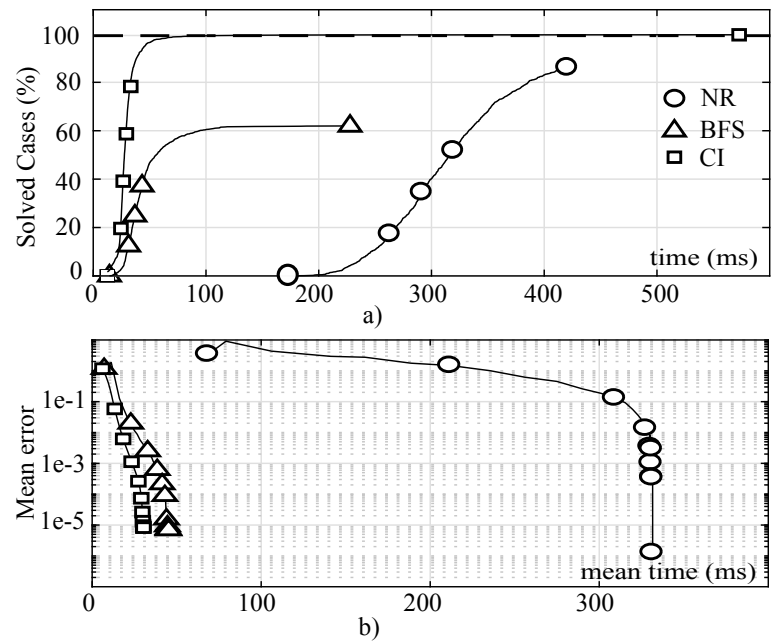

Fig. 15. Convergence analysis of the three algorithms with the case A. a) Percentage of solved cases with the time. b) Average error propagation

The link connecting substations S6 (blue line) and S5 (orange line) is unidirectional, so it only has negative power (from destination to source) when the voltage drop across the link (voltage in source node minus voltage in destination node) is lower than $-10 \mathrm{~V}$. As it can be observed in Fig. 13 d), the voltage drop is positive most of the time, so the link remains blocked.

Fig. 13 e) represents the voltage across the link connecting the substations S4 (green line) and S4 (red line) and the power in its source node (S4 green line). The link is a bidirectional link with deadband. The voltage drop lies within a band of around $\pm 100 \mathrm{~V}$ (referred to the source node). The destination to source power flow (negative) is activated when the voltage drop falls below $-20 \mathrm{~V}$. The source to destination power flow (positive) is activated when the voltage drop is higher than $40 \mathrm{~V}$. The performance of the first train departing from S1 of red line is depicted in Figs. 13 f), g) and h). Fig. 13 f) contains the catenary voltage (in black) and the power reference (in red). Positive power represents traction. The state of charge of the accumulator and the charging and discharging power are represented in Fig. $13 \mathrm{~g}$ ). It can be observed how the charging and discharging power saturate to their maximum in several occasions. Finally, Fig. 13 h) shows the burnt power in the rheostatic system of the train (in black) and the non-supplied power (in red). It must be remarked that there is burnt power in three occasions even when the accumulator is not full. In such occasions the accumulator is being charged at its maximum rate and the train overvoltage protection is activated. It can be observed as well, that the overcurrent protection of the train is activated just once during the first half an hour when the catenary voltage drops below $2650 \mathrm{~V}$.

\section{B. Algorithm performance analysis}

The performance of the the algorithm was tested with multiple realistic and random cases and compared with the speed and convergence of other algorithms like NewtonRaphson (NR) and Backward/Forward Swept (BFS). In this subsection, these parameters are analysed for the abovedescribed case study and a simplification of it, in which all substations are bidirectional. In Table III] a comparison between the minimum time, maximum time and average time invested in solving each instant, as well as the percentage of instants successfully solved can be observed for the case study (Case A) and its simplification with all substations bidirectional (Case B). As it can be observed, the three algorithms achieve a convergence of $100 \%$ with the case B and the BFS algorithm is the fastest one with $0.013 \mathrm{~ms}$ per instant. In this case, the NR is the slowest algorithm. With the case A (adding non-bidirectional substations), the proposed algorithm is the fastest one with an average time of $0.030 \mathrm{~ms}$ per instant, i.e., nearly ten times faster than the NR. The speed of the BFS is similar to the speed of the CI, but the convergence achieved by the BFS is only $63 \%$. The NR convergence rate is arround $90 \%$. The figures of the proposed algorithm (CI) in Table III were obtained considering a damping factor of 0.2 . In Table II , the effect of the damping factor over the speed and the convergence rate is analysed for the proposed algorithm. In this case, there is a band between 0.15 and 0.30 in which $100 \%$ of convergence is achieved with similar average convergence time. The algorithm is faster near the borders of the stable interval, but the speed difference is not very high (just $0.003 \mathrm{~ms}$ ) and the probability of obtaining unsolved cases increases. As it was stated, the selection of the damping factor is critical, since out of this band the convergence drops drastically. Usually a set of random tests is carried out in order to obtain the best damping factor for a specific network. Further improvements of the algorithm will consider a dynamic selection of the damping factor. High values of the damping factor increase the computation time since they increase significantly the oscillations during the iterative process.

Finally, in the Fig. 15, a graphical comparison of the three algorithms is depicted. Fig. 15 a) represent the percentage of solved cases against the time with the three algorithms. Considering that an interval of 30 minutes is studied and one 
case represent one second, there are a total of 1800 cases. As it can be observed, CI algorithm solves $95 \%$ of the cases in less than $50 \mathrm{~ms}$ investing more than $500 \mathrm{~ms}$ in solving the $5 \%$ of the cases. These last set of cases are those in which most of the substations are blocked at the same time. In Fig. 15b), the propagation of the mean error with the time is represented.

\section{CONCLUSIONS}

In the paper, a comprehensive set of general models, able to simulate most of the devices present in modern DC railways is presented. The models were developed in a simple but accurate way and a formulation to embed such models in current injection power flow algorithm is proposed. The compatibility of the proposed mathematical models and formulation was tested in a complex and realistic case study containing all possible devices: unidirectional and bidirectional substations with and without deadband, links connecting different DC levels, off-board and on-board accumulators and trains with overvoltage and overcurrent protection. Catenary free segments were also considered in the simulation. The models were implemented in a modified current injection based power flow algorithm with damping factor. The proposed algorithm shows a stable and robust performance when comparing with other algorithms like Newton-Raphson and Backward/Forward Swept.

\section{REFERENCES}

[1] S. Talukdar and R. Koo, "The analysis of electrified ground transportation networks," Power Apparatus and Systems, IEEE Transactions on, vol. 96, no. 1, pp. 240-247, 1977.

[2] T. Suzuki, "DC power-supply system with inverting substations for traction systems using regenerative brakes," Electric Power Applications, IEE Proceedings B, vol. 129, no. 1, pp. 18-26, January 1982.

[3] Y. Cai, M. Irving, and S. Case, "Iterative techniques for the solution of complex dc-rail-traction systems including regenerative braking," Generation, Transmission and Distribution, IEE Proceedings, vol. 142, no. 5, pp. 445-452, 1995.

[4] C. Pires, S. Nabeta, and J. Cardoso, "ICCG method applied to solve dc traction load flow including earthing models," Electric Power Applications, IET, vol. 1, no. 2, pp. 193-198, 2007.

[5] P. Arboleya, G. Diaz, and M. Coto, "Unified AC/DC power flow for traction systems: A new concept," Vehicular Technology, IEEE Transactions on, vol. 61, no. 6, pp. 2421-2430, July 2012.

[6] M. Coto, P. Arboleya, and C. Gonzalez-Moran, "Optimization approach to unified $\mathrm{AC} / \mathrm{DC}$ power flow applied to traction systems with catenary voltage constraints," International Journal of Electrical Power \& Energy Systems, vol. 53, no. 0, pp. $434-441,2013$.

[7] Z. Tian, S. Hillmansen, C. Roberts, P. Weston, N. Zhao, L. Chen, and M. Chen, "Energy evaluation of the power network of a DC railway system with regenerating trains," IET Electrical Systems in Transportation, vol. 6, no. 2, pp. 41-49, 2016.

[8] R. A. Jabr and I. Dzafic, "Solution of DC railway traction power flow systems including limited network receptivity," IEEE Transactions on Power Systems, vol. PP, no. 99, pp. 1-1, 2017.

[9] P. Arboleya, "Heterogeneous multiscale method for multirate railway traction systems analysis," IEEE Transactions on Intelligent Transportation Systems, vol. PP, no. 99, pp. 1-6, 2016.

[10] P. Arboleya, B. Mohamed, C. Gonzalez-Moran, and I. El-Sayed, "Bfs algorithm for voltage-constrained meshed dc traction networks with nonsmooth voltage-dependent loads and generators," IEEE Transactions on Power Systems, vol. 31, no. 2, pp. 1526-1536, March 2016.

[11] B. Mohamed, P. Arboleya, and C. Gonzalez-Moran, "Modified current injection method for power flow analysis in heavy-meshed DC railway networks with non-reversible substations," IEEE Transactions on Vehicular Technology, vol. PP, no. 99, pp. 1-1, 2017.

[12] R. R. Pecharroman, A. Lopez-Lopez, A. P. Cucala, and A. FernandezCardador, "Riding the rails to DC power efficiency: Energy efficiency in dc-electrified metropolitan railways." IEEE Electrification Magazine, vol. 2, no. 3, pp. 32-38, Sept 2014.
[13] P. Arboleya, M. Coto, C. Gonzalez-Moran, and R. Arregui, "On board accumulator model for power flow studies in dc traction networks," Electric Power Systems Research, vol. 116, no. Supplement C, pp. 266 - 275, 2014.

[14] P. Arboleya, P. Bidaguren, and U. Armendariz, "Energy is on board: Energy storage and other alternatives in modern light railways," IEEE Electrification Magazine, vol. 4, no. 3, pp. 30-41, Sept 2016.

[15] S. de la Torre, A. J. Sánchez-Racero, J. A. Aguado, M. Reyes, and O. Martínez, "Optimal sizing of energy storage for regenerative braking in electric railway systems," IEEE Transactions on Power Systems, vol. 30, no. 3, pp. 1492-1500, May 2015.

[16] B. Mellitt, Z. S. Mouneimne, and C. J. Goodman, "Simulation study of dc transit systems with inverting substations," IEE Proceedings $B$ Electric Power Applications, vol. 131, no. 2, pp. 38-50, March 1984.

[17] C. Mayet, P. Delarue, A. Bouscayrol, E. Chattot, and J. N. Verhille, "Comparison of different emr-based models of traction power substations for energetic studies of subway lines," IEEE Transactions on Vehicular Technology, vol. 65, no. 3, pp. 1021-1029, March 2016.

[18] V. Gelman, "Energy storage that may be too good to be true: Comparison between wayside storage and reversible thyristor controlled rectifiers for heavy rail," IEEE Vehicular Technology Magazine, vol. 8, no. 4, pp. 7080, Dec 2013.

[19] M. Chymera, A. Renfrew, M. Barnes, and J. Holden, "Modeling electrified transit systems," Vehicular Technology, IEEE Transactions on, vol. 59, no. 6, pp. 2748-2756, 2010.

[20] T. Ratniyomchai, S. Hillmansen, and P. Tricoli, "Recent developments and applications of energy storage devices in electrified railways," IET Electrical Systems in Transportation, vol. 4, no. 1, pp. 9-20, March 2014.

[21] C. Mayet, L. Horrein, A. Bouscayrol, P. Delarue, J.-N. Verhille, E. Chattot, and B. Lemaire-Semail, "Comparison of different models and simulation approaches for the energetic study of a subway," Vehicular Technology, IEEE Transactions on, vol. 63, no. 2, pp. 556-565, Feb 2014.

[22] J. R. Martí, H. Ahmadi, and L. Bashualdo, "Linear powerflow formulation based on a voltage-dependent load model," IEEE Transactions on Power Delivery, vol. 28, no. 3, pp. 1682-1690, July 2013.

[23] Y. Tzeng, R. Wu, and N. Chen, "A detailed RL fed bridge converter model for power flow studies in industrial AC/DC power systems," Industrial Electronics, IEEE Transactions on, vol. 42, no. 5, pp. 531$538,1995$.

[24] F. Milano, Power System Modelling and Scripting. Springer, 2010.

[25] J. Aguado, A. J. Sanchez-Racero, and S. de la Torre, "Optimal operation of electric railways with renewable energy and electric storage systems," IEEE Transactions on Smart Grid, vol. PP, no. 99, pp. 1-1, 2017.

[26] D. Chen and L. Xu, "Autonomous de voltage control of a de microgrid with multiple slack terminals," IEEE Transactions on Power Systems, vol. 27, no. 4, pp. 1897-1905, Nov 2012.

[27] T. R. Oliveira, W. W. A. Gonçalves-Silva, and P. F. Donoso-Garcia, "Distributed secondary level control for energy storage management in dc microgrids," IEEE Transactions on Smart Grid, vol. 8, no. 6, pp. 2597-2607, Nov 2017.

[28] L. Abrahamsson and L. Soder, "Fast estimation of relations between aggregated train power system data and traffic performance," Vehicular Technology, IEEE Transactions on, vol. 60, no. 1, pp. 16-29, 2011.

[29] C. A. Charalambous, I. Cotton, and P. Aylott, "Modeling for preliminary stray current design assessments: The effect of crosstrack regeneration supply," IEEE Transactions on Power Delivery, vol. 28, no. 3, pp. 18991908, July 2013.

[30] Y.-S. Tzeng and C.-H. Lee, "Analysis of rail potential and stray currents in a direct-current transit system," Power Delivery, IEEE Transactions on, vol. 25, no. 3, pp. 1516-1525, July 2010.

[31] E. R. Ramos, A. G. Exposito, and G. A. Cordero, "Quasi-coupled threephase radial load flow," IEEE Transactions on Power Systems, vol. 19, no. 2, pp. 776-781, May 2004.

[32] I. Kocar and J. S. Lacroix, "Implementation of a modified augmented nodal analysis based transformer model into the backward forward sweep solver," IEEE Transactions on Power Systems, vol. 27, no. 2, pp. 663-670, May 2012.

[33] P. Yan and A. Sekar, "Analysis of radial distribution systems with embedded series facts devices using a fast line flow-based algorithm," IEEE Transactions on Power Systems, vol. 20, no. 4, pp. 1775-1782, Nov 2005.

[34] R. A. Jabr and I. Dzafic, "Sensitivity-based discrete coordinate-descent for volt/var control in distribution networks," IEEE Transactions on Power Systems, vol. 31, no. 6, pp. 4670-4678, Nov 2016. 